\title{
A Framework of Cloud Model Similarity-Based Quality Control Method in Data-Driven Production Process
}

\author{
Sheng Hu $\mathbb{D}^{1,2}$ Shuanjun Song $\mathbb{D}^{1,2}$ and Wenhui Liu ${ }^{1,2}$ \\ ${ }^{1}$ School of Mechanical and Electrical Engineering, Xi'an Polytechnic University, Xi'an, China \\ ${ }^{2} X i$ 'an Key Laboratory of Modern Intelligent Textile Equipment, Xi'an Polytechnic University, Xi'an, China \\ Correspondence should be addressed to Sheng Hu; husheng@xpu.edu.cn
}

Received 29 October 2019; Revised 30 March 2020; Accepted 23 April 2020; Published 18 May 2020

Academic Editor: Ruben Specogna

Copyright (c) 2020 Sheng Hu et al. This is an open access article distributed under the Creative Commons Attribution License, which permits unrestricted use, distribution, and reproduction in any medium, provided the original work is properly cited.

Considering the problem that the process quality state is difficult to analyze and monitor under manufacturing big data, this paper proposed a data cloud model similarity-based quality fluctuation monitoring method in data-driven production process. Firstly, the randomness of state fluctuation is characterized by entropy and hyperentropy features. Then, the cloud pool drive model between quality fluctuation monitoring parameters is built. On this basis, cloud model similarity degree from the perspective of maximum fluctuation border is defined and calculated to realize the process state analysis and monitoring. Finally, the experiment is conducted to verify the adaptability and performance of the cloud model similarity-based quality control approach, and the results indicate that the proposed approach is a feasible and acceptable method to solve the process fluctuation monitoring and quality stability analysis in the production process.

\section{Introduction}

Intelligent manufacturing can discover the potential laws of the production process through data mining technology, and the intelligent decision model can be built by using this rule to realize green and intelligent manufacturing [1]. With the increasing use of personalization in large-, medium-, and small-batch manufacturing processes, the quality of products is also getting higher and higher expected requirements $[2,3]$. In an actual production process, there are many influencing factors that affect the process fluctuation and exhibit temporal correlation, multidimensional coupling, dynamics, and high reliability $[4,5]$. Once the stability of fluctuation state is not timely evaluated, it will lead the abnormal state to continue to transfer, accumulate, and grow to ultimately result in the process abnormalities [6, 7]. Thus, how to quantitative characterize the quality fluctuation and timely monitor the process state is the key link to guarantee the production process stability and product quality qualified, which will be of great significance to improve production efficiency and product quality, reduce energy and resource consumption, and finally realize the intelligent and green development in the production process.

To address this issue, this paper mainly focuses on the process fluctuation and proposes a data cloud model similarity-based quality-monitoring approach in the production process. The idea of process fluctuation cloud model construction, cloud model similarity calculation, and quality fluctuation monitoring of current process are successively adopted. The basic idea is to use the cloud model theory to model the process fluctuation, and the cloud model similarity is introduced to measure the fluctuation degree. Specifically, it includes three works. Firstly, the cloud digital features are calculated based on the data acquisition of key influence variables. Secondly, the triad is integrated to establish the process fluctuation cloud model, which is used to characterize process fluctuation. Finally, in order to monitor the process fluctuation amplitude, the cloud model similarity theory is introduced to calculate the similarity degree, which is used to monitor the current process fluctuation and the degree of internal stability by judging the amount of similarity. In this way, the dynamic fluctuation of process quality is obtained to support the production process quality 
monitoring and evaluation. It hopes that the proposed method will provide some useful suggestions for enhancing the process stability and product quality in the production process. The rest of this paper is organized as follows: Section 2 briefly states the related works and motivations. In Section 3 , the theoretical modeling of the data cloud model drivenbased quality-monitoring approach is reviewed, which includes the quality fluctuation characterization based on the data cloud model and process quality monitoring based on cloud model similarity analysis. Section 4 provides the experiments and results. Finally, the conclusions are drawn and some recommendations for future research are given in Section 5.

\section{Related Works and Motivations}

In this section, a literature review is summarized in the field of process state modeling and monitoring as well as the monitor model based on related algorithms.

2.1. Process Quality Modeling and Monitoring. Process quality modeling and monitoring is the crucial work to analyze the process stability of quality fluctuation in intelligent production process $[8,9]$. In this field, several quality modeling methods have been put forward to guarantee the process fluctuation in the sustain-stable state. The statistical quality control $[10,11]$ and state-space model $[12,13]$ were successively proposed to analyze fluctuation state and quality errors in production process. With the development of artificial intelligence and information technology and its application in the manufacturing field, domestic and foreign scholars constantly explore the application of data-driven methods in process quality control and improvement technology [14], including the quality monitoring [15], capability performance analysis [16], and the change point technology [17, 18]. Yazid et al. [19] established an adaptive support vector machine-based temperature monitoring and surface quality evaluation model in continuous casting process. Online test results show an ideal implementable application effect. In order to improve process quality, Chang et al. [20] analyze the processes' capability performance with multiple characteristics through the accuracy and precision index by applying the quality level concept of the six-sigma model. They developed a statistical hypothesis testing mode to evaluate production department effectiveness of implemented improvements by using the mathematical programming. Nestic et al. [21] proposed a modeling method to assess and optimize the quality of production process, which provides the theoretical basis for improvement of the production process quality.

In the field of process-monitoring aspects, there have been remarkable works on the quality stability monitoring for statistical process control $[22,23]$. After nearly a hundred years of development and application, SPC has played an important role in the quality control and improvement of the production process. Aslam et al. [24] monitored the process quality by using attribute data combined with variable data to build the mixed control chart to achieve the goal. They discuss that the proposed mixed control chart provides quick and efficient indication than the existing method once the process is uncontrolled. Shongwe Graham [25] reported that only if an out-of-control event falls on one side of the center line, the modified side-sensitive (MSS) synthetic chart will report signals. Wang et al. [26] proposed a novel economic profit model for designing a fully adaptive control chart for monitoring continuous production processes, which are allowed to vary to maximize quality-related profit rather than minimize costs.

Through the above analysis, it can be seen that the existed research studies have been explored some useful ideas in quality state monitoring. Due to the strong uncertainty in the production process, the existing qualitymonitoring methods still have many problems in the recognition of accuracy. How to use quality data from the perspective of multidimensional data flow to model and monitor quality fluctuations in the production process is still a key issue to be solved in the field of quality control.

2.2. Monitor Model Based on Related Algorithms. Appropriate algorithms-based evaluation model is useful for improving quality control effect. Generally, various history steady-state data are adopted to construct the monitor model and the real-time data are acquitted to analyze and monitor the degree of stability for the current process state. Many scholars have conducted the process state monitoring and analysis in-depth research. The monitoring and diagnosis of process-operating performance [27] and corresponding control charts' monitoring [28] were proposed. For instance, Yazid et al. [19] proposed a surface quality evaluation and temperature-monitoring model for continuous casting process. The adaptive filtering-based ASVR technology provides an excellent evaluation result in the steel industry. Amdouni et al. [29] proposed a variable sampling interval control chart to monitor the variation coefficient in short production runs, and the die-casting hotchamber actual data are adopted to illustrate the effective of the proposed approach. Although various modeling methods for the production process have relative advantages and disadvantages, various modeling methods are not good or bad, just to see which method is more suitable for a specific occasion. Due to the multidimensional time-varying, nonlinear, strong coupling, and uncertainty characteristics of modern production processes, it is more difficult to accurately model the process [30, 31]. Fortunately, modeling methods based on quality state data show unique advantages in solving the above problems. In addition, the computer-monitoring system of the modern production process records and stores a large amount of production process data and product quality data, providing a basis and guarantee for data-driven quality state control technology.

These studies mainly hold the steps that by obtaining sufficient sample data, the quality control model is constructed to monitor and evaluate the quality fluctuation. However, it is necessary to further study how to use data mining and analysis technology to describe and mine the relationship between quality data. On the other hand, how to 
effectively use these data to model quality fluctuation and explore the quality fluctuation mechanism in the production process remains to be further studied. If the whole product manufacturing process is regarded as a comprehensive dynamic system which is open to the outside world, by using acquired quality data, the process quality fluctuation can be quantitative description and characterization. This makes it possible to use data mining techniques to study fluctuation mechanisms and the quality-monitoring method.

In the actual application process, the cloud model theory, as a typical representative of data modeling methods, is widely used for modeling the relationship between the lifetime of samples and testing stress levels [30], water quality assessment [31], and other fields. The main idea of the cloud model method is that by extracting the characteristics of state data related to the formation of quality states and calculating the data cloud drops, a cloud model capable of characterizing the state of things is constructed to evaluate the state level.

Based on the above analysis, this article transplants the superiority of the cloud model theory into the field of quality control. Its basic ideal is that by combining the certainty of production process fluctuation and the randomness of fluctuation states, a qualitative and quantitative mutual mapping cloud model of the process quality state is constructed, which is used to characterize the quality fluctuation characteristics. Based on this, the current process fluctuations are compared with the fluctuation threshold of the stable process state to determine whether the current process fluctuations meet the desired stability criteria. Thus, the proposed method in this paper mainly concentrates on solving the following problems and exploring a data cloud model driven-based quality-monitoring approach in production process.

(1) Real-time monitoring is the nature of dynamic process quality control. Due to the factors such as resource, stability, environment, and equipment, the formation process of product quality is real-time dynamic change. So, the production process fluctuation model should be built as a state measure for quality fluctuation monitoring.

(2) Calculating the fluctuation degree of state change is a critical step for process quality fluctuation monitoring. In fact, how to build the quality fluctuation measure strategy is the link. Therefore, the cloud model similarity analysis is used to evaluate the degree of internal stability by judging the amount of similarity.

\section{Theoretical Modeling for Process Quality Monitoring}

The idea of data cloud model similarity analysis includes three key technologies, which integrates to implement the process monitor. Figure 1 shows the proposed modeling logical flow.

It can be seen from in Figure 1 that there are three execution steps. Firstly, cloud digital feature triad is calculated to establish the process fluctuation cloud model and regarded as a standard for fluctuation monitoring. Secondly, in order to use the cloud model to monitor quality fluctuation of the production process, the cloud model similarity is defined and similarity measure matrix is calculated by real time to monitor the process quality fluctuation state. Finally, as for current quality fluctuation, the intersection overlap area of the cloud model boundary curve is calculated to accomplish the quality fluctuation monitoring.

The proposed approach has its special characteristics listed as follows: (1) the method considers the process quality fluctuation as a quality data flow along with the process ongoing, and the quality state is tracked by establishing the quality data cloud model between state variables. (2) The cloud model similarity is introduced to define the fluctuation amplitude, which is used to calculate the similarity of cloud models for different quality states. (3) The relationship between the fluctuation degree of production process and the cloud similarity degree for different process states is applied to build the quality cloud similarity-based monitoring model to calculate the quality fluctuation amount to achieve the quality monitor.

Based on the above characteristics, the proposed method shows some advantages listed as follows: (1) by extracting the influenced state factors, the cloud model is established from the level of digital features to qualitatively characterize process quality fluctuations, which could timely and dynamically track changes in quality fluctuations and avoid the drawbacks of statistical quality modeling. (2) The cloud model similarity principle is adopted to describe the similarity for different quality fluctuations, and the quality fluctuation monitoring model is built to reveal the evolution trend of abnormal quality. In summary, the proposed cloud model-driven approach realizes the quality state monitoring of the process stability, which may be suitable for the timely monitor quality state in the field of production process quality control.

\subsection{Quality Fluctuation Characterization Using Cloud Model Theory. The quality fluctuation modeling of the production process refers to construct a qualitative and quantitative mapping by combining the fluctuation certainty and the state quality randomness. For the purpose, the constructed quality cloud model can reflect the state characteristics of quality fluctuations in the production process, which realizes the purpose of cloud model characterization and state monitoring for different quality fluctuation levels. Thus, the quality fluctuation cloud model is defined as follows.}

Definition 1. The quality fluctuation cloud model $I X_{n}=\left(x_{1}, x_{2}, \ldots, x_{m}\right) o$, where, $m$ is the quality state dimension and $n$ is the quality sample data size. The fluctuation degree of the quality state can be described through each quality feature, which refers to the cloud drops $x_{1}, x_{2}, \ldots, x_{m}$. The quantified cloud drop $x_{m}$ reflects the quantification degree of feature, the number of cloud drops, and degree of quantification directly affects the monitoring 


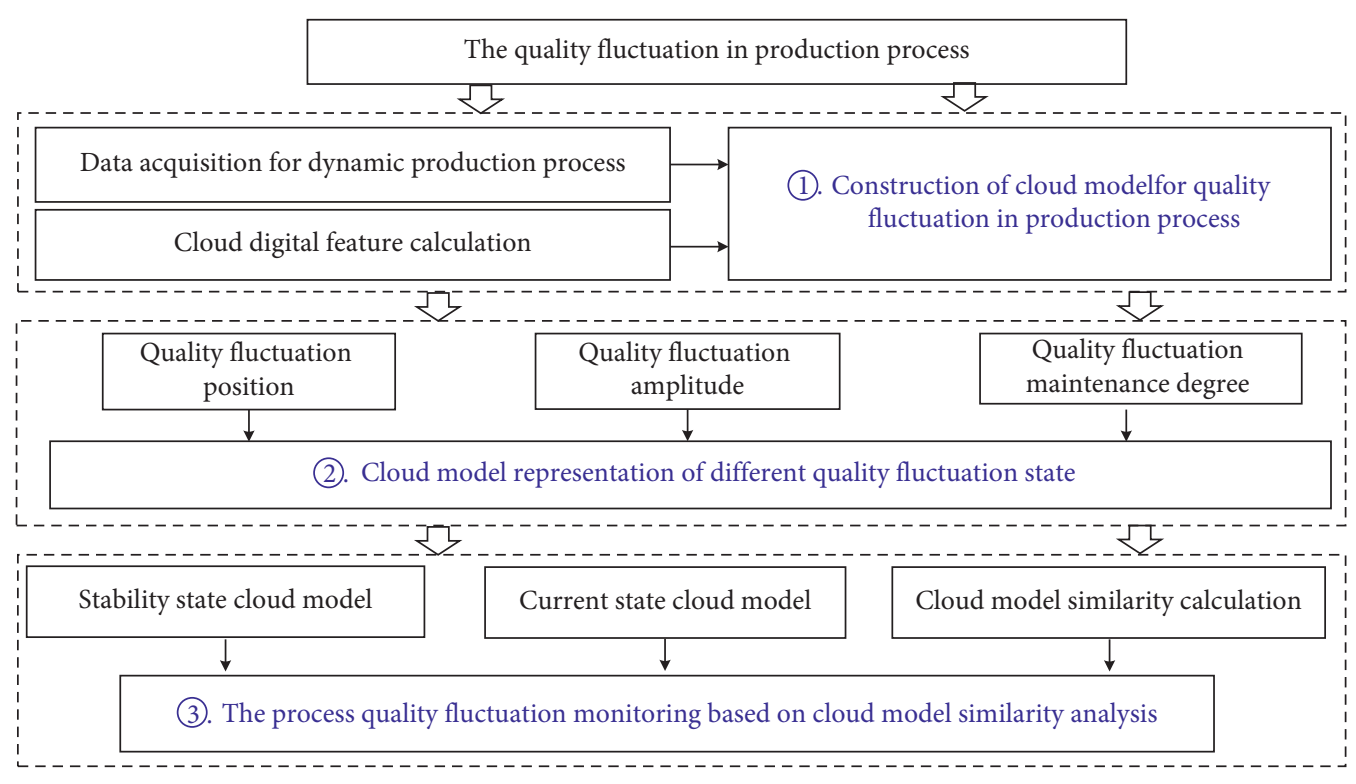

FIGURE 1: Logical flow of data cloud model driven-based process quality monitoring.

accuracy of quality state fluctuation. And the more the number of cloud drops, the higher the degree of quantification, which results in the higher accuracy of quality status monitoring effect.

Thus, the quality-monitoring cloud model is a triple model that composed of process quality state data to generate a set of cloud-drop features, which is used to reflect the level of quality state fluctuations. The expectation Ex is the mean of information space, which is the index that best describes the fluctuation state and trajectory of the corresponding indicator. The entropy En is the measure of uncertainty of qualitative concepts, which is determined by the ambiguity and randomness of qualitative concepts. The superentropy $\mathrm{He}$ is a measure of the uncertainty for entropy, which reflects the thickness of cloud and the degree of deviation of each indicator data in the quality fluctuation space. This paper uses the cloud generator to convert the quality analysis data of the production process into a cloud model corresponding to the quality fluctuation. Thus, the cloud model construction process for quality monitoring of production process is built as follows:

(1) As for the $m$-dimensional quality state monitoring sample sequence $X=\left\{x_{1 j}, x_{2 j}, \ldots, x_{n j}\right\}^{T}$, the mean and variance are calculated as follows:

$$
\left\{\begin{array}{l}
\bar{X}=\left(\bar{x}_{1}, \bar{x}_{2}, \ldots, \bar{x}_{p}\right)^{T}, \\
s_{i j}=\frac{1}{N} \sum_{i=1}^{N}\left(x_{i j}-\bar{x}_{j}\right)\left(x_{i j}-\bar{x}_{j}\right)^{T},
\end{array}\right.
$$

where $\bar{x}_{j}=(1 / N) \sum_{i=1}^{N} x_{i j}(j=1,2, \ldots, m)$.

(2) Calculating the Ex, En, and $\mathrm{He}$, respectively, we get

$$
\left\{\begin{array}{l}
\mathrm{Ex}=\bar{X}, \\
\mathrm{En}=\sqrt{\frac{\pi}{2} \times \frac{1}{N} \sum_{i=1}^{N}\left|x_{i}-\mathrm{Ex}\right|,} \\
\mathrm{He}=\sqrt{\frac{1}{N-1} \sum_{i=1}^{N}\left(\bar{x}_{i}-\mathrm{Ex}\right)^{2}-(\mathrm{En})^{2}} .
\end{array}\right.
$$

(3) The multidimensional inverse cloud generator is used to generate a central cloud drop $\left(\left(x_{1}, x_{2}, \ldots, x_{m}\right), u_{c}\right)$, which is a cloud drop in quality fluctuation space.

(4) Steps 1-3 are repeated until a sufficient number of desired cloud drops have been generated. Thus, the cloud drop group is obtained to characterize process quality fluctuations of the quality state space, which, respectively, corresponds to three numerical features in the quality fluctuation cloud model.

3.1.1. Quality Fluctuation Position. It is used to characterize the relative position of the current state to the stable state of the production process, which is divided into three categories that are fluctuation position center, fluctuation position left, and fluctuation position right. This indicator is determined by the quality characteristics of the production process.

3.1.2. Quality Fluctuation Amplitude. It is used to characterize the amplitude which the current state deviates from the relative position of the center of the steady state process, 
which is divided into three categories: the fluctuation position center, fluctuation position left, and fluctuation position right. This indicator is determined by the quality characteristics of the production process. It is divided into three categories that are maintained in good condition, state remains normal, and state remains poor. This indicator reflects the fluctuation magnitude in process quality. The more stable the current process is, the smaller fluctuation the quality becomes.

3.1.3. Quality Fluctuation Maintenance Degree. It is used to characterize the maintenance degree of the quality fluctuation state over a period of time, which is divided into three categories that are state maintains good, normal, and poor. This indicator has relations with fluctuation factors and changes in processing equipment state.

3.2. Process Quality Monitoring Based on Cloud Model Similarity Analysis. This section is used to elaborate how to quantitatively monitor the quality fluctuations of current process in real time in detail. Considering the similarity strategy of the cloud model theory, the cloud model similarity is defined and the similarity measure matrix is calculated for process quality; thus, the real-time monitor quality level of the process is achieved. The process quality monitor based on cloud model similarity analysis is shown in Figure 2.

As shown in Figure 2, the cloud model similaritybased quality-monitoring strategy contains two parts. Firstly, by constructing the sample data cloud pool for quality fluctuation, the similarity measure of the cloud model for current fluctuation state and the cloud model for stable state levels is analyzed to obtain the similarity measure matrix. Then, the area of the overlap region based on the boundary curve of the quality state cloud model is used to characterize the similarity of different quality states in production process; thus, quality fluctuations in current processes are real-time monitored through the state-similar clouds analysis.

Definition 2. Cloud model similarity degree (CMSD) refers to proximity degree which the current fluctuation level of the quality state to the data cloud model corresponding to the zero-fluctuation ideal stable-state quality level.

The corresponding zero-fluctuation ideal stable process state cloud set $\mathrm{ICS}_{t}$ and current fluctuation process state cloud $\mathrm{CCS}_{t}$ of the production process quality in the $t$ period are expressed as

$$
\mathrm{ICS}_{t}=\left[\left(\mathrm{Ex}_{1 t}, \mathrm{En}_{1 t}, \mathrm{He}_{1 t}\right)^{I},\left(\mathrm{Ex}_{2 t}, \mathrm{En}_{2 t}, \mathrm{He}_{2 t}\right)^{I}, \ldots,\left(\mathrm{Ex}_{n t}, \mathrm{En}_{n t}, \mathrm{He}_{n t}\right)^{I}\right],
$$

$\mathrm{CCS}_{t}=\left[\left(\mathrm{Ex}_{1 t}, \mathrm{En}_{1 t}, \mathrm{He}_{1 t}\right)^{C},\left(\mathrm{Ex}_{2 t}, \mathrm{En}_{2 t}, \mathrm{He}_{2 t}\right)^{C}, \ldots,\left(\mathrm{Ex}_{n t}, \mathrm{En}_{n t}, \mathrm{He}_{n t}\right)^{C}\right]$.
According to the digital characteristics of the quality state cloud model, the cloud model similarity $\mathrm{CMSD}_{t}$ can be obtained as

$$
\mathrm{CMSD}_{t}=\frac{S_{C^{I} \cap C^{C}}}{S_{C^{I} \cup C^{C}}}=\frac{f\left(\mathrm{Ex}_{1 t}, \mathrm{En}_{1 t}, \mathrm{He}_{1 t}\right)^{I} \cap f\left(\mathrm{Ex}_{1 t}, \mathrm{En}_{1 t}, \mathrm{He}_{1 t}\right)^{C}}{f\left(\mathrm{Ex}_{1 t}, \mathrm{En}_{1 t}, \mathrm{He}_{1 t}\right)^{I} \cup f\left(\mathrm{Ex}_{1 t}, \mathrm{En}_{1 t}, \mathrm{He}_{1 t}\right)^{C^{\prime}}}
$$

where $\mathrm{CMSD}_{t}$ is the cloud model similarity value at the time $t$ in the production process. It represents the ratio of volume for the public area $S$ to the total area $S_{0}$ of the cloud model, which can be obtained by multiple integral of the cloud model boundary curve. $f$ is the maximum boundary curve of the quality cloud model, which is calculated by the following formula:

$$
f(\mathrm{Ex}, \mathrm{En}, \mathrm{He})=\exp \left[-\frac{(x-\mathrm{Ex})^{2}}{2(3 \mathrm{He}+\mathrm{En})^{2}}\right] .
$$

Based on the above definition and analysis, the range of the CMSD for the quality cloud model is $[0,1]$ and $\mathrm{CMSD}_{t}=1$ means that the process state is in a zero-fluctuation ideal stable level. For actual production process, due to the influence of human-machine environment and other factors existed along with process ongoing, $\mathrm{CMSD}_{t}$ should be less than $1 . \mathrm{CMSD}_{t}=0$ means that the process state is in a completely abnormal fluctuation level. For this characteristic, the index CMSD could be used as a similarity measure between the current quality and stable-state cloud model in production process. It can be seen that the similarity degree between current quality and stable quality state increases along with the value of $\mathrm{CMSD}_{t}$ increases. Specifically, the larger the $\mathrm{CMSD}_{t}$ is, the closer the process quality is to the zero-fluctuation ideal stable-state quality. Conversely, the smaller the $\mathrm{CMSD}_{t}$ is, the farther the process state deviates from the zero-fluctuation ideal stable state, where the more likely the abnormal quality state occurs. Without loss of generality, for a binary variable group of the production process, the CMSD should be expressed as the volume ratio of the space boundary of the cloud model which surrounded by the three-dimensional image. Thus, according to the calculation result of cloud similarity for different quality fluctuations, it could realize the goal of real monitoring of the state fluctuation stability in production process.

\section{Experiments and Results}

4.1. Datasets Description. In order to demonstrate the application prospects of the proposed approach, this section uses an experiment case, which a cotton spinning process dataset is adopted to validate the effectiveness of the proposed model. During its production process, the fiber length and fiber uniformity are the two key quality features that have an important influence on the breaking strength of the yarn. It should be strictly controlled. Therefore, in order to effectively monitor the quality fluctuation of the yarnbreaking strength, 3000 sample data for fiber length and fiber uniformity in a stable process state are collected for one yarn processing in the cotton spinning process. Based on these samples, the cloud digital feature triplet is calculated to build 


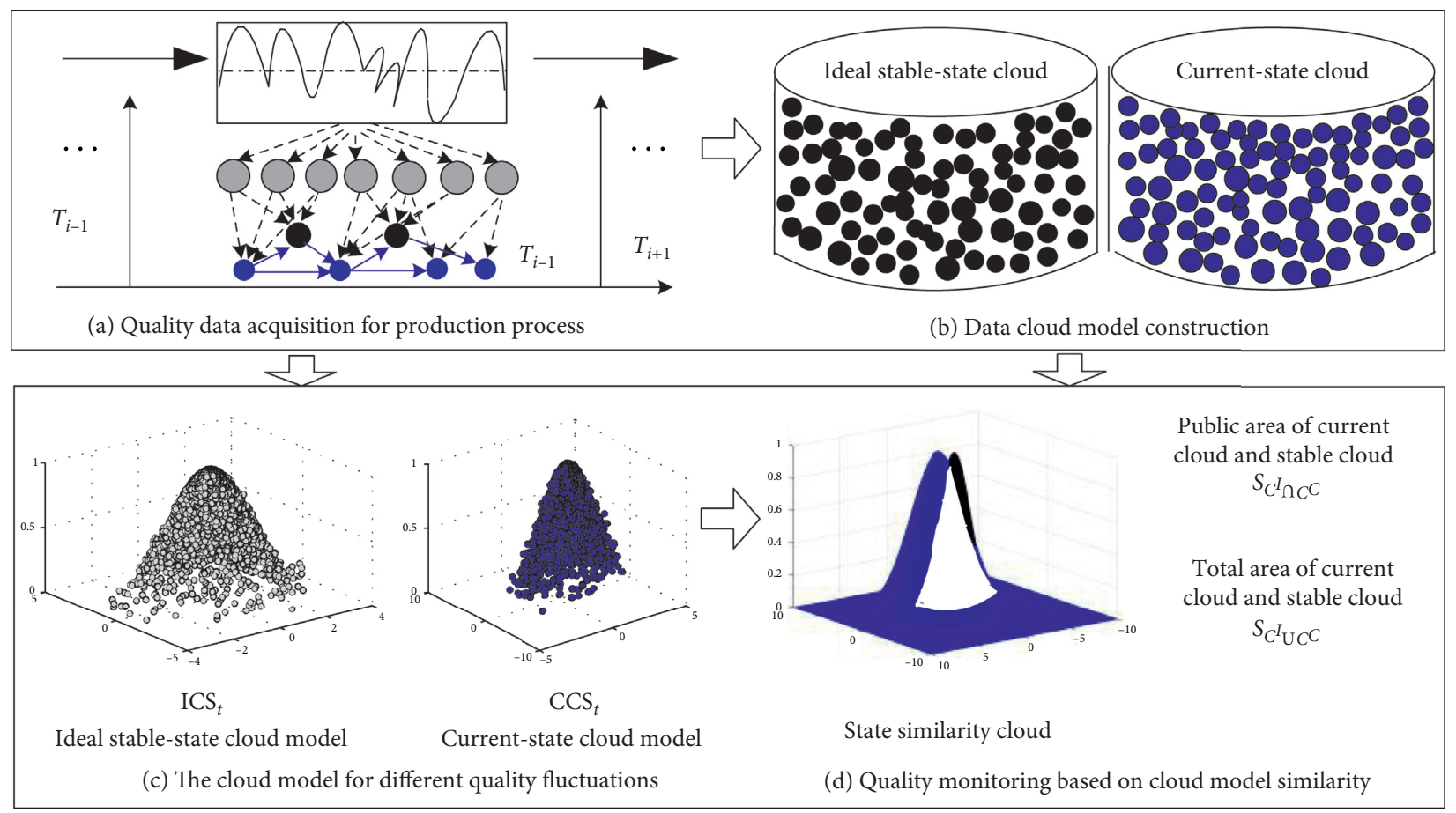

Figure 2: The cloud model similarity-based quality-monitoring strategy.

the cloud model for this ideal stable-state, and the cloud model triplet is $\{(0,0),(1,1),(0.1,0.1)\}$, which is regarded as the benchmark for zero-fluctuation ideal stable-state. For the process of building cloud models for other fluctuation cases, the numerical simulation is used to obtain other three abnormal states. Specifically, other forms of datasets with different degrees of fluctuation are obtained by adding three different varying degrees of fluctuation amplitude perturbations on the basis of the stable-state quality dataset, which is used to calculate the cloud digital characteristics to obtain the corresponded cloud model. The triplet set of the cloud digital feature for these process fluctuation states are $\{(3,3),(1.2,1.2),(0.1,0.1)\}, \quad\{(-3,-3),(1.2,1.2),(0.1,0.1)\}$, and $\{(0,0),(1.5,1.5),(0.3,0.3)\}$, and it is the foundation of constructing the quality fluctuation cloud model and cloud model similarity-based quality fluctuation monitor.

4.2. Cloud Model Construction. In order to construct the cloud model for the four different fluctuation states, the multidimensional inverse cloud generator is started, followed the cloud model building steps in Section 3.1 to generate desired cloud drops. In the actual modeling process, the number of quality data cloud-drops for each fluctuation state is 3000 . Based on the generated cloud-drop datasets, four different types of cloud digital feature combinations for the quality state and their corresponding quality fluctuation types are shown in Table 1. According to the $4 * 3000$ samples for four types of cloud-drop data, the cloud models for each quality fluctuation state in cotton spinning process are constructed and shown in Figure 3.

As shown in Figure 3, it can be seen in Figure 3(a) that the space center of the fluctuating cloud model is near the expectation value and the thickness of the cloud model is small, which indicates that the deviation of state data for the process quality in the cloud model space is close to the expected value of the stable-state process. Thus, the quality state mode is regarded as the standard to analyze and monitor other process fluctuation states. As shown in Figures 3(b) and 3(c), the fluctuating centers are deviated from the ideal position and diverge on other sides of the fluctuation center, which reveals the process quality is in an uncontrolled mode. Compared with the above circumstances, Figure 3(d) shows more thickness of space distribution than that of Figure 3(a), this means the process fluctuation states are in a controlled trend but with a poor maintain degree, which may easily cause abnormal process fluctuations. Furthermore, in order to real-time monitor the process fluctuation, the cloud model similarity-based quality-monitoring strategy is further applied to quantitative analyze the similarity degree of the quality state for each quality fluctuation mode in next subsection.

\subsection{Quality Fluctuation Monitoring Based on Cloud Model} Similarity. Without loss of generality, the proposed cloud model similarity method is implemented to qualify fluctuation monitoring for the above stable quality state (Figure 3(a)) and the abnormal fluctuation state (Figure 3(b)). State similar cloud and fluctuation cloud boundary are shown in Figure 4.

As shown in Figure 4(a), the space fluctuation position of the current quality cloud model has a certain degree of deviation from the stable quality space fluctuation position. Thus, by calculating the public area and total area volume composed of the wave boundary 
TABLE 1: Quality fluctuation types corresponding to different cloud model parameters.

\begin{tabular}{lcc}
\hline No. & Cloud model parameters & Quality fluctuation types on different cloud digital features \\
\hline 1 & $\{(0,0),(1,1),(0.1,0.1)\}$ & Median fluctuation, small fluctuation amplitude, and state remains good \\
2 & $\{(3,3),(1.2,1.2),(0.1,0.1)\}$ & Left-side fluctuation, medium fluctuation amplitude, and state remains general \\
3 & $\{(-3,-3),(1.2,1.2),(0.1,0.1)\}$ & Right-side fluctuation, medium fluctuation amplitude, and state remains general \\
4 & $\{(0,0),(1.5,1.5),(0.3,0.3)\}$ & Median fluctuation, large fluctuation amplitude, and state remains poor \\
\hline
\end{tabular}

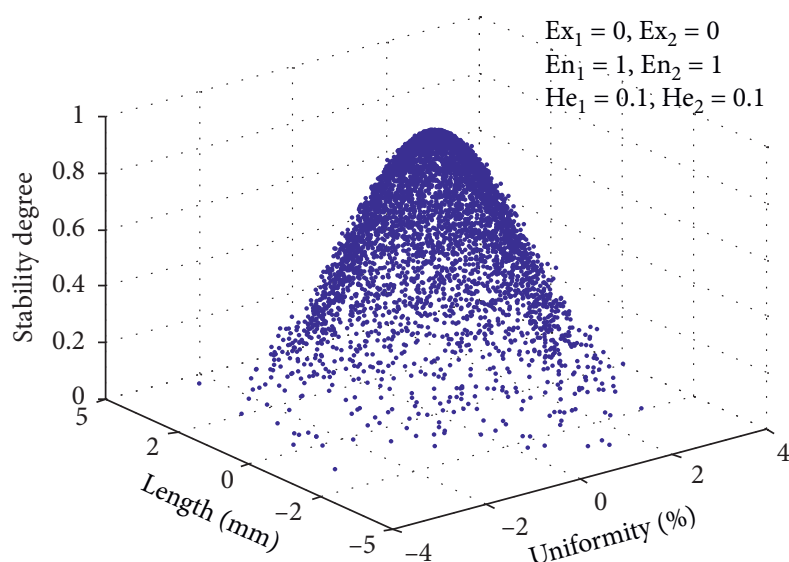

(a)

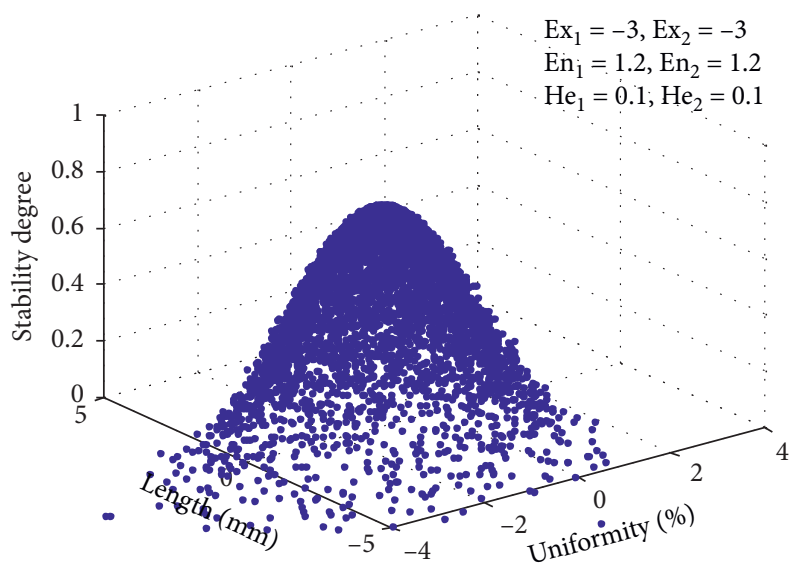

(c)

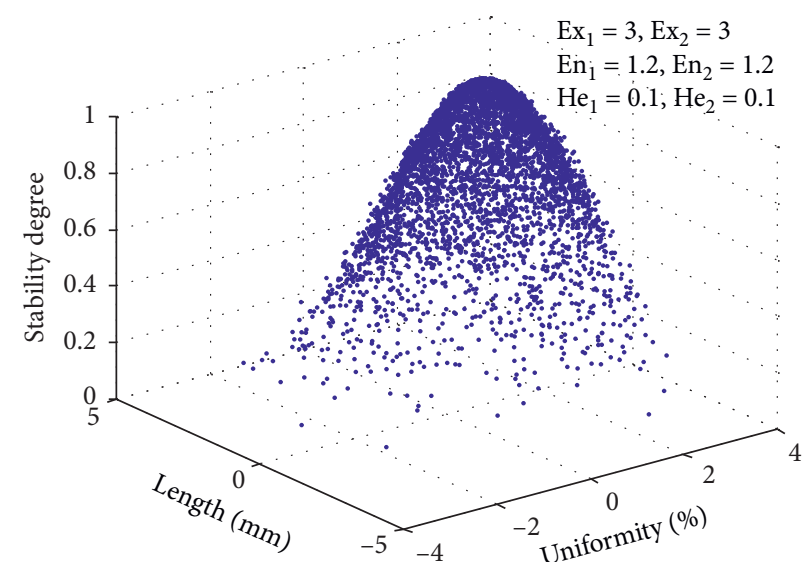

(b)

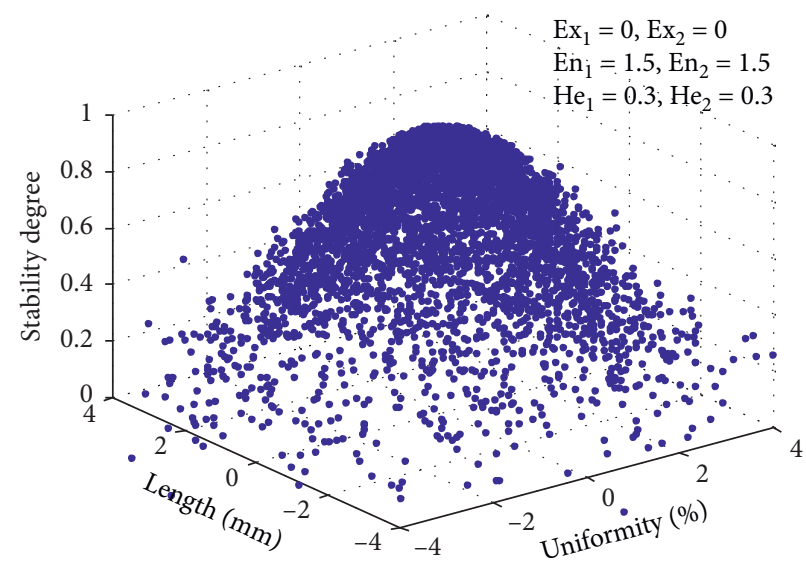

(d)

Figure 3: Cloud model for different quality fluctuations in cotton spinning process. (a) Median fluctuation, small fluctuation amplitude, and state remains good; (b) left-side fluctuation, medium fluctuation amplitude, and state remains general; (c) right-side fluctuation, medium fluctuation amplitude, and state remains general; (d) median fluctuation, large fluctuation amplitude, and state remains poor.

surface for cloud drop collection, the cloud model similarity with the quality state is shown in Figure 4(b). It shows that the current process quality deviates from the fluctuation trajectory of the stable cloud model, which easily causes the occurrence of the abnormal quality state. Some measures should be taken to prevent the continued spread of abnormal quality.

What should be explained that this paper mainly focuses on the could model similarity degree-based quality monitor for production process, this proposed method has certain requirements on the continuity of the process state, so it could not be suitable for abnormal quality fluctuation monitoring for a short time. And it is not only limited for process quality monitoring but also for being suitable for other problems of production area such as capacity assessment and cloud prediction for machining quality.

4.4. Discussions on Proposed Method. As for the proposed approach, there are two key points for process quality monitoring, which are quality fluctuation cloud model construction and CMSD for quality stability monitoring. In summary, the theoretical value of applying the method proposed in this paper to actual process quality monitoring is listed as follows: 


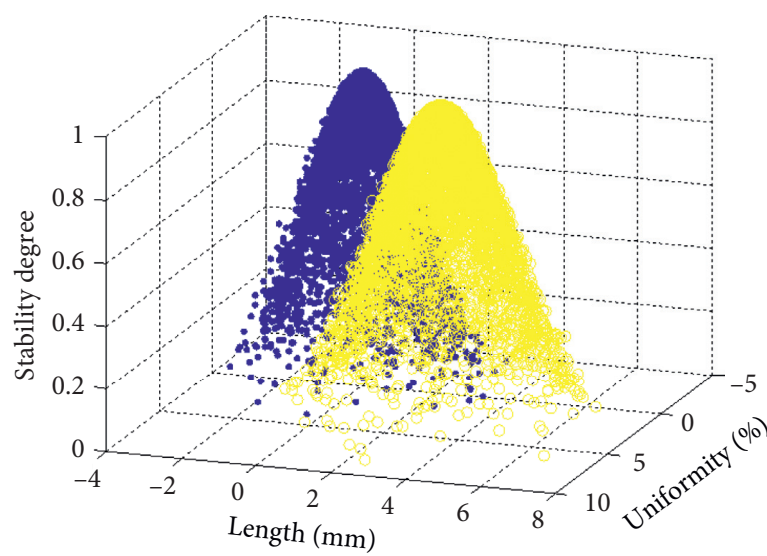

Stable cloud

Current cloud

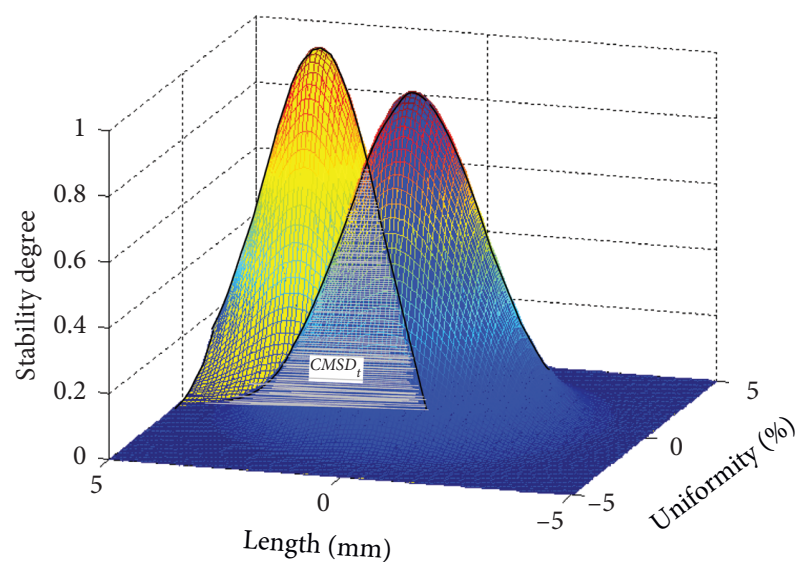

A Stable cloud boundary

Current cloud boundary

Public area

(a)

(b)

FIgURE 4: The state similar cloud and fluctuation cloud boundary for different quality fluctuations. (a) State similar cloud and (b) fluctuation cloud boundary.

(1) The quality fluctuation cloud model adapted to different processes is constructed to analyze the overall quality fluctuation, which uses the digital features of the cloud model. It better integrates the ambiguity and randomness in the qualitative and quantitative conversion of process quality and quality state parameters.

(2) The cloud model similarity method is introduced to calculate the similarity of cloud models with different quality states. By comparing the similarity degree between the cloud model of the current process state and the cloud model of the zero-fluctuation ideal stable state, the stability monitoring of the current process state is realized.

Actually, in order to assure the proposed cloud model monitor method can be more rigorous and accurate for applied into actual industry production process possibility, much more repeatedly experiments should be tested on the proposed monitor model for its stability and reliability, such as test on larger samples or in different datasets, and even further quantitative theoretical proof process should be carried out as a support. Here, more quantitative theoretical proof process for the cloud model similarity-based quality control method is not provided. Although the proof process is just from the angle of qualitative level, to some extent, the proposed quality-control method implies some advantages and is sufficient to draw the theoretical value above. The authors will try to further explore the proposed approach in the future and perfect it.

\section{Conclusions and Future Works}

Aiming at the problem of quality fluctuation modeling and monitoring in production process, this paper proposes a process quality-monitoring method based on data cloud model similarity. The method quantifies the degree of fluctuation of process quality by entropy and superentropy, which reflects the process fluctuation variability and the quality state randomness. The contributions are drawn as follows:

(1) Using the digital feature of the cloud model to analyze the quality state fluctuations as a whole, it better integrates the ambiguity and randomness in the quantitative conversion of processing quality and quality state parameters, and the digital features of the constructed cloud model can reflect the fluctuation characteristic of the quality state. At the same time, the constructed quality cloud model could represent the process fluctuation degree effectively.

(2) Cloud model similarity technology is introduced to solve the similarity of quality cloud models with different fluctuation levels, in which it uses cloud model digital features to describe the similarity of the quality fluctuation state. The current quality volatility level is monitored based on the similarity calculation results. The monitoring results of the above cases show that the proposed cloud model similarity method can effectively capture the difference of quality state fluctuation information at different process stages.

However, what should be stated is that this approach is still in an early test stage and may have some application limitation. The proposed cloud model monitoring method gives the corresponding relationship between the CMSD and the stability degree of the process state just from a qualitative level. In order to ensure the rigor of the theoretical method, it is necessary to carry out a strict theoretical proof of the relationship between CMSD and the quality stability degree. Moreover, this method has certain requirements on the 
continuity of the process state, so it could not detect the abnormal quality fluctuation of the process state in a short time, which is also the limitation of the method.

Future works will focus on two parts listed as follows: one is to deeply carry out theoretical research on the cloud model theory in the field of quality control, using multiple cases to verify the feasibility of the cloud model monitoring method and expand its application scope and the other is to explore the abnormal quality fluctuation warning method to predict and contain the quality state abnormal fluctuation, in which the multiple change point theory is considered to be introduced into quality warning for abnormal fluctuation.

\section{Data Availability}

The data used in the submitted paper are based on actual simulation data of the cotton-spinning process dataset. Readers can access the data supporting the conclusions of the study by the simulation condition related in the case simulation of the paper.

\section{Conflicts of Interest}

The authors declare that they have no conflicts of interest.

\section{Acknowledgments}

This work was supported by the Scientific Research Program Funded by Xi'an Scientific and Technological Innovation Platform Construction Project/Key Laboratory Construction Project (no. 2019220614SYS021CG043), Doctoral Research Startup Fund Project of XPU (grant no. BS201834), and Shaanxi Provincial Education Department (program no. 19JK0234).

\section{References}

[1] J. Ding, T. Chai, H. Wang, J. Wang, and X. Zheng, “An intelligent factory-wide optimal operation system for continuous production process," Enterprise Information Systems, vol. 10, no. 3, pp. 286-302, 2016.

[2] L. Shi, L. Newnes, S. Culley, and B. Allen, "Modelling, monitoring and evaluation to support automatic engineering process management," Proceedings of the Institution of $\mathrm{Me}$ chanical Engineers, Part B: Journal of Engineering Manufacture, vol. 232, no. 1, pp. 17-31, 2018.

[3] J. Wang, L. Zhang, L. Duan, and R. X. Gao, "A new paradigm of cloud-based predictive maintenance for intelligent manufacturing," Journal of Intelligent Manufacturing, vol. 28, no. 5, pp. 1125-1137, 2017.

[4] T.-F. Li, S. Hu, Z.-Y. Wei et al., "A framework for diagnosing the out-of-control signals in multivariate process using optimized support vector machines," Mathematical Problems in Engineering, vol. 2013, Article ID 494626, 9 pages, 2013.

[5] M. O. Besenhard, O. Scheibelhofer, K. Francois, M. Joksch, and B. Kavsek, "A multivariate process monitoring strategy and control concept for a small-scale fermenter in a PAT environment," Journal of Intelligent Manufacturing, vol. 29, no. 7, pp. 1501-1514, 2018.

[6] G. Zhang, Y. Ran, Y. Wang, and T. Gao, "Composite error prediction of multistage machining processes based on error transfer mechanism," The International Journal of Advanced Manufacturing Technology, vol. 76, no. 1-4, pp. 271-280, 2015.

[7] B. Kamsu-Foguem, F. Rigal, and F. Mauget, "Mining association rules for the quality improvement of the production process," Expert Systems with Applications, vol. 40, no. 4, pp. 1034-1045, 2013.

[8] G. Wang, Y. Zhang, C. Liu, Q. Xie, and Y. Xu, "A new tool wear monitoring method based on multi-scale PCA," Journal of Intelligent Manufacturing, vol. 30, no. 1, pp. 113-122, 2019.

[9] L.-Y. Ouyang, C.-H. Hsu, and C.-M. Yang, "A new process capability analysis chart approach on the chip resistor quality management," Proceedings of the Institution of Mechanical Engineers, Part B: Journal of Engineering Manufacture, vol. 227, no. 7, pp. 1075-1082, 2013.

[10] W. H. Woodall, "The use of control charts in health-care and public-health surveillance," Journal of Quality Technology, vol. 38, no. 2, pp. 89-104, 2006.

[11] D. Rahali, P. Castagliola, H. Taleb, and M. B. C. Khoo, "Evaluation of Shewhart time-between-events-and-amplitude control charts for several distributions," Quality Engineering, vol. 31, no. 2, pp. 240-254, 2019.

[12] J. Liu, J. Shi, and S. J. Hu, "Quality-assured setup planning based on the stream-of-variation model for multi-stage machining processes," IIE Transactions, vol. 41, no. 4, pp. 323-334, 2009.

[13] A. Bazdar, R. B. Kazemzadeh, and S. T. A. Niaki, "Variation source identification of multistage manufacturing processes through discriminant analysis and stream of variation methodology: a case study in automotive industry," Journal of Engiering Research, vol. 3, no. 2, pp. 1-14, 2015.

[14] J.-B. Yu and L.-F. Xi, "A neural network ensemble-based model for on-line monitoring and diagnosis of out-of-control signals in multivariate manufacturing processes," Expert Systems with Applications, vol. 36, no. 1, pp. 909-921, 2009.

[15] J. Shi and S. Zhou, "Quality control and improvement for multistage systems: a survey," IIE Transactions, vol. 41, no. 9, pp. 744-753, 2009.

[16] B. Jiang, X. Zhu, D. Huang, and R. D. Braatz, "Canonical variate analysis-based monitoring of process correlation structure using causal feature representation," Journal of Process Control, vol. 32, pp. 109-116, 2015.

[17] M. Ayoubi, R. B. Kazemzadeh, and R. Noorossana, "Change point estimation in the mean of multivariate linear profiles with no change type assumption via dynamic linear model," Quality and Reliability Engineering International, vol. 32, no. 2, pp. 403-433, 2016.

[18] S. Hu, L. Zhao, Y. Yao, and R. Dou, "A variance change point estimation method based on intelligent ensemble model for quality fluctuation analysis," International Journal of Production Research, vol. 54, no. 19, pp. 5783-5797, 2016.

[19] L. D. L. Yazid, B. Salah, B. M. Seghir, and B. Jurgen, "Adaptive support vector machine-based surface quality evaluation and temperature monitoring: application to billet continuous casting process," The International Journal of Advanced Manufacturing Technology, vol. 67, no. 9-12, pp. 2063-2073, 2013.

[20] T.-C. Chang, K.-J. Wang, and K.-S. Chen, "Capability performance analysis for processes with multiple characteristics using accuracy and precision," Proceedings of the Institution of Mechanical Engineers, Part B: Journal of Engineering Manufacture, vol. 228, no. 5, pp. 766-776, 2014.

[21] S. Nestic, M. Stefanovic, A. Djordjevic, S. Arsovski, and D. Tadic, "A model of the assessment and optimisation of production process quality using the fuzzy sets and genetic 
algorithm approach," European Journal of Industrial Engineering, vol. 9, no. 1, pp. 77-99, 2015.

[22] M.-H. Shu and H.-C. Wu, "Manufacturing process performance evaluation for fuzzy data based on loss-based capability index," Soft Computing, vol. 16, no. 1, pp. 89-99, 2012.

[23] M. Sekar, J. Srinivas, K. R. Kotaiah et al., "Stability analysis of turning process with tailstock-supported workpiece," The International Journal of Advanced Manufacturing Technology, vol. 43, no. 9-10, pp. 862-871, 2009.

[24] M. Aslam, M. Azam, N. Khan, and C.-H. Jun, “A mixed control chart to monitor the process," International Journal of Production Research, vol. 53, no. 15, pp. 4684-4693, 2015.

[25] S. C. Shongwe and M. A. Graham, "A modified side-sensitive synthetic chart to monitor the process mean," Quality Technology \& Quantitative Management, vol. 15, no. 3, pp. 328-353, 2018.

[26] R.-F. Wang, X. Fu, J.-C. Yuan, and Z.-Y. Dong, "Economic design of variable-parameter X-Shewhart control chart used to monitor continuous production," Quality Technology \& Quantitative Management, vol. 15, no. 1, pp. 106-124, 2018.

[27] J. F. MacGregor and T. Kourti, "Statistical process control of multivariate processes," Control Engineering Practice, vol. 3, no. 3, pp. 403-414, 1995.

[28] M. S. F. Nezhad and S. T. A. Niaki, “A Max-EWMA approach to monitor and diagnose faults of multivariate quality control processes," The International Journal of Advanced Manufacturing Technology, vol. 68, no. 9-12, pp. 2283-2294, 2013.

[29] A. Amdouni, P. Castagliola, H. Taleb, and G. Celano, "A variable sampling interval Shewhart control chart for monitoring the coefficient of variation in short production runs," International Journal of Production Research, vol. 55, no. 19, pp. 5521-5536, 2017.

[30] W. Zhang, S. Liu, B. Sun, Y. Liu, and M. Pecht, "A cloud model-based method for the analysis of accelerated life test data," Microelectronics Reliability, vol. 55, no. 1, pp. 123-128, 2015.

[31] D. Wang, D. Zeng, V. P. Singh et al., "A multidimension cloud model-based approach for water quality assessment," Environmental Research, vol. 149, pp. 113-121, 2016. 